\title{
ENDOMORPHISMS OF AN EXTREMAL ALGEBRA
}

\author{
HERBERT KAMOWITZ AND DENNIS WORTMAN
}

(Communicated by John B. Conway)

\begin{abstract}
Let $E a[-1,1]$ denote the extremal algebra on $[-1,1]$ as defined in Bonsall and Duncan, Numerical ranges. II. We show that every nonzero endomorphism $T$ on $E a[-1,1]$ has the form $T f(x) \rightarrow f(A x+B)$ where $A$ and $B$ are real and $|A|+|B| \leq 1$. Further, the endomorphism $T$ is an automorphism if, and only if, $B=0$ and $A=1$ or -1 , while $T$ is a nonzero compact endomorphism if, and only if, $T: f(x) \rightarrow f(B)$ for some $B$ in $[-1,1]$. Also included in this note are several results related to compact endomorphisms of regular commutative semisimple Banach algebras.
\end{abstract}

In this note, we determine the endomorphisms of the extremal algebra $E a[-1,1]$ for whose definition we follow the notations of $[1, \S 24]$. Let $C$ denote the complex field, $M(C)$ the set of finite regular Borel measures on $C$ and $M^{\omega}(C)$ the set of measures $\mu \in M(C)$ for which $\int_{C} e^{|\operatorname{Re} \lambda|} d|\mu|(\lambda)<\infty$. Then $E a[-1,1]=$ $\left\{f \mid f(x)=\int_{C} e^{x \lambda} d \mu(\lambda)\right.$ for some $\left.\mu \in M^{\omega}(C),-1 \leq x \leq 1\right\}$. With norm defined by $\|f\|=\inf \left\{\int_{C} e^{|\operatorname{Re} \lambda|} d|\mu|(\lambda) \mid \mu \in M^{\omega}(C)\right.$ with $\left.f(x)=\int_{C} e^{x \lambda} d \mu(\lambda)\right\}, E a[-1,1]$ is a regular commutative semisimple Banach algebra which satisfies a strong Ditkin condition $[1,4]$. Further, the maximal ideal space of $E a[-1,1]$ is $[-1,1]$. The algebra $E a[-1,1]$ is called the extremal algebra for $[-1,1]$, a name derived from a property it possesses relative to the study of numerical ranges of elements in complex unital Banach algebras. $E a[-1,1]$ is generated by the hermitian element $u$, where $u(x)=x$ for $x \in[-1,1]$. We remark that $\left\|e^{i t u}\right\|=1$ for all real $t[1,4]$.

Let $D[-1,1]$ denote the Banach space of entire functions $\phi$ such that $\|\phi\|=$ $\sup \left\{|\phi(\lambda)| e^{-|\operatorname{Re} \lambda|} \mid \lambda \in C\right\}<\infty$. Then $D[-1,1]$ is isometrically isomorphic to the dual space of $E a[-1,1]$ under the correspondence $\phi \rightarrow \Phi_{\phi}$ where $\Phi_{\phi}(f)=$ $\int_{C} \phi(\lambda) d \mu(\lambda)$ for any representation $f(x)=\int_{C} e^{x \lambda} d \mu(\lambda)[1$, p. 59].

It is easy to show that if $A$ and $B$ are real numbers with $|A|+|B| \leq 1$, then $T: E a[-1,1] \rightarrow E a[-1,1]$, given by $T f(x)=f(A x+B)$, for $x \in[-1,1]$, is an endomorphism of $E a[-1,1]$. Our primary purpose in this paper is to show that there are no other nonzero endomorphisms of $E a[-1,1]$.

MAIN THEOREM. Let $T$ be a nonzero endomorphism of $E a[-1,1]$. Then $T$ has the form $T f(x)=f(A x+B)$ for some real numbers $A$ and $B$ with $|A|+|B| \leq 1$.

This result resembles the Beurling-Helson Theorem which states that every nonzero endomorphism of the group algebra $L^{1}(-\infty, \infty)$ is generated by a linear change of variable. Indeed, the proof of our result follows in a similar manner to that of the Beurling-Helson Theorem presented in [3, pp. 217-218].

Received by the editors November 30, 1987 and, in revised form, February 29, 1988. Presented to the Society, January 8, 1988.

1980 Mathematics Subject Classification (1985 Revision). Primary 47B38, 46J99, 47A12. 
The results in this paper arose out of a study of compact endomorphisms of regular Banach algebras. In many cases, such as $C(X), L^{1}(G)$ and $C^{n}\left([0,1]^{m}\right)$, if the maximal ideal space is connected, then, when the algebra has an identity 1 , every nonzero compact endomorphism $T$ of the algebra has the form $T f=\hat{f}\left(x_{0}\right) 1$ for some $x_{0}$ in the maximal ideal space, while, when the algebra has no identity, there are no nonzero compact endomorphisms of the algebra. We conjectured that this is true in general. Professor John Duncan suggested $E a[-1,1]$ as a possible counterexample, and, in the course of examining it, we were led to the Main Theorem.

Subsequently, we have found an example of a regular commutative semisimple unital Banach algebra with a connected maximal ideal space and a nonzero compact endomorphism which does not have the form $f \rightarrow \hat{f}\left(x_{0}\right) 1$. For such an example, let

$$
B=\left\{f \in C^{\infty}[0,1] \mid\|f\|=\sum_{n=0}^{\infty} \frac{\left\|f^{(n)}\right\|_{\infty}}{(n !)^{2}}<\infty\right\}
$$

with pointwise addition and multiplication. Then $B_{0}$ is a regular commutative semisimple unital Banach algebra whose maximal ideal space is the interval $[0,1]$. The map $T: f(x) \rightarrow f(x / 2)$ is a compact endomorphism.

However, for $E a[-1,1]$ and, in fact, for any semisimple Banach algebra generated by 1 and a hermitian element $u$ whose spectrum is a closed interval $[a, b]$, every nonzero compact endomorphism $T$ has the form $T f=\hat{f}\left(x_{0}\right) 1$ for some $x_{0} \in[a, b]$, which is the maximal ideal space of the algebra. Indeed, let $B$ be the algebra generated by 1 and $u$. If $T$ is a nonzero compact endomorphism of $B$, then there exists $g \in B$ with $T u=g$ and so, for each $f \in B$ and all $x \in[a, b], T F^{\wedge}(x)=\hat{f}(\hat{g}(x))$. Clearly, $\hat{g}:[a, b] \rightarrow[a, b]$. Let $x_{0}$ be a fixed point of $\hat{g}$, and, for each positive integer $n$, let $f_{n} \in B$ be defined by $f_{n}=\left(\left(1+e^{-i x_{0}} e^{i u}\right) / 2\right)^{n}$. Since $\left\|e^{i k u}\right\|_{B}=1$ for all integers $k,\left\{f_{n}\right\}$ is a bounded sequence in $B$, and since $T$ is a compact operator, there exists an $F \in B$ and a subsequence $\left\{n_{k}\right\}$ of the positive integers such that $T f_{n_{k}} \rightarrow F$ as $k \rightarrow \infty$. This implies that, for each $x \in[a, b], T f_{n_{k}}^{\wedge}(x)=\left(\left(1+e^{-i x_{0}} e^{i \hat{g}(x)}\right) / 2\right)^{n_{k}} \rightarrow$ $\hat{F}(x)$. Hence,

$$
\hat{F}(x)= \begin{cases}1 & \text { if } \hat{g}(x)=x_{0}+2 m \pi \text { for some integer } m, \\ 0 & \text { otherwise }\end{cases}
$$

However, $\hat{F}$ is continuous so that $\hat{g}(x)=x_{0}+2 m \pi$ for some integer $m$, and, since $\hat{g}$ is continuous and $\hat{g}\left(x_{0}\right)=x_{0}$, we conclude that $\hat{g}(x)=x_{0}$ for all $x \in[a, b]$. That is, for each $f \in B, T f^{\wedge}(x)=\hat{f}\left(x_{0}\right) 1$ for all $x \in[a, b]$.

We proceed to the main part of the paper, first stating the following lemma from which Lemma 1 follows easily.

LEMMA [2, LEMMA 1.6]). Let $[a, b]$ be a compact interval in $R$ and $\psi \in$ $\mathscr{D}(] a, b[)$ and $k \in C^{p}([a, b])$ with

$$
0<C_{1} \leq\left|k^{\prime}(x)\right|+\left|k^{\prime \prime}(x)\right|+\cdots+\left|k^{(p)}(x)\right| \leq C_{2},
$$

if $x \in[a, b]$, where $C_{1}$ and $C_{2}$ are constants and $p$ is a positive integer. Then there exists a constant $C$ not depending on $k$, such that

$$
\left|\int_{a}^{b} e^{i s k(x)} \psi(x) d x\right| \leq C s^{-1 / p}
$$


for every $s>0$.

LEMMA 1. If $g$ is a nonlinear polynomial function, then

$$
\lim _{n \rightarrow \infty} \sup _{s \in R}\left|\int_{-1}^{1} e^{i s x} e^{-i n g(x)} d x\right|=0 \text {. }
$$

With this lemma, we show that a nonlinear polynomial does not induce an endomorphism of $E a[-1,1]$. The special case $g(x)=x^{2}$ is a consequence of Theorem 27.5 of $[1$, p. 81].

LEMMA 2. If $g:[-1,1] \rightarrow[-1,1]$ is a nonlinear polynomial function, then $g$ does not induce an endomorphism of $E a[-1,1]$.

PROOF. Suppose a nonlinear polynomial function $g$ induces an endomorphism $T$ of $E a[-1,1]$. Then, if $u$ is the identity function on $[-1,1], u(x)=x$, we have $T u=g$. For each $n>0$, let $\phi_{n}(x)=\int_{-1}^{1} e^{z x} e^{-i n g(x)} d x$. Then $\phi_{n}$ is an entire function, and since $x \operatorname{Re} z \leq|\operatorname{Re} z|$ for $-1 \leq x \leq 1$,

$$
\begin{aligned}
\sup _{z \in C}\left|\phi_{n}(z)\right| e^{-|\operatorname{Re} z|} & =\sup _{z \in C}\left|\int_{-1}^{1} e^{z x} e^{-|\operatorname{Re} z|} e^{-i n g(x)} d x\right| \\
& \leq \sup _{z \in C} \int_{-1}^{1} e^{x \operatorname{Re} z-|\operatorname{Re} z|} d x \\
& \leq 2 .
\end{aligned}
$$

Therefore, for each $n, \phi_{n}(z) \in D[-1,1]$, and so, for each $z \in C$,

$$
\Phi_{\phi_{n}}\left(e^{z u}\right)=\phi_{n}(z)=\int_{-1}^{1} e^{z x} e^{-i n g(x)} d x .
$$

(The first equality above is Theorem 24.5 in $[1$, p. 59].)

Since $\left\{e^{z u}\right\}$ generates $E a[-1,1]$, we have, for all $f \in E a[-1,1]$,

$$
\Phi_{\phi_{n}}(f)=\int_{-1}^{1} f(x) e^{-i n g(x)} d x .
$$

It follows from the Phragmén-Lindelöf Theorem that

$$
\begin{aligned}
\left\|\Phi_{\phi_{n}}\right\| & =\left\|\phi_{n}\right\|_{D[-1,1]} \\
& =\sup _{z \in C}\left|\phi_{n}(z)\right| e^{-|\operatorname{Re} z|} \\
& =\sup _{s \in R}\left|\phi_{n}(i s)\right| \\
& =\sup _{s \in R}\left|\int_{-1}^{1} e^{i(s x-n g(x))} d x\right| .
\end{aligned}
$$

Furthermore, if we let $f_{n}=T\left(e^{i n u}\right)=e^{i n g(x)}$, then $f_{n} \in E a[-1,1]$, and, from equation (1),

$$
\Phi_{\phi_{n}}\left(f_{n}\right)=\int_{-1}^{1} e^{i n g(x)} e^{-i n g(x)} d x=2 .
$$

Since $g$ induces the endomorphism $T$ and $\left\|e^{i n u}\right\|=\left\|e^{i n x}\right\|_{\infty}=1$, we have

$$
2=\left\|\Phi_{\phi_{n}}\left(T\left(e^{i n u}\right)\right)\right\| \leq\left\|\Phi_{\phi_{n}}\right\|\|T\| .
$$


Therefore,

$$
2 \leq\|T\| \sup _{s \in R}\left|\int_{-1}^{1} e^{i(s x-n g(x))} d x\right| .
$$

By Lemma 1, if $g$ is a nonlinear polynomial function, then the right side of this last inequality approaches 0 as $n$ gets large. This contradiction shows that no polynomial of degree $\geq 2$ induces an endomorphism of $E a[-1,1]$.

The proof of the next lemma closely parallels that of the Beurling-Helson Theorem in [3, pp. 217-219].

LEMMA 3. Suppose $T$ is an endomorphism of Ea[-1,1] and $T f(x)=f(g(x))$ for all $f \in E a[-1,1], x \in[-1,1]$. Then there exists an interval $I \subset[-1,1]$ on which $g$ is a linear function.

ProOF. Let $T$ and $g$ be as described in the hypotheses, and let $T^{*}$ be the adjoint of $T$. Changing the notation from the proof of Lemma 2, we set $\phi_{\xi}(\lambda)=e^{\lambda \xi}$, for $-1 \leq \xi \leq 1$. Then $\Phi_{\phi_{\xi}}$ is point evaluation at $\xi$, i.e.,

$$
\Phi_{\phi_{\xi}}(f)=\int_{C} e^{\xi \lambda} d \mu_{f}(\lambda)=f(\xi)
$$

for all $f \in E a[-1,1]$. Moreover,

$$
T^{*} \Phi_{\phi_{\xi}}(f)=\Phi_{\phi_{\xi}}(T f)=T f(\xi)=f(g(\xi))=\Phi_{\phi_{g(\xi)}}(f)
$$

for all $\xi \in[-1,1]$.

Hence, for all $a_{k} \in C$ and $\xi_{k} \in[-1,1]$,

and so

$$
T^{*}\left(\sum a_{k} \Phi_{\phi_{\xi_{k}}}\right)=\sum a_{k} \Phi_{\phi_{g\left(\xi_{k}\right)}}=\Phi_{\sum a_{k} \phi_{g\left(\xi_{k}\right)}}
$$

$$
\begin{aligned}
\left\|T^{*}\left(\sum a_{k} \Phi_{\phi_{\xi_{k}}}\right)\right\| & =\left\|\Phi_{\sum a_{k} \phi_{g\left(\xi_{k}\right)}}\right\|_{D[-1,1]} \\
& =\sup _{\lambda \in C}\left|\sum a_{k} e^{g\left(\xi_{k}\right) \lambda}\right| e^{-|\operatorname{Re} \lambda|} \\
& =\sup _{x \in R}\left|\sum a_{k} e^{i g\left(\xi_{k}\right) x}\right|,
\end{aligned}
$$

again from applying the Phragmén-Lindelöf Theorem.

Using Kronecker's Theorem [3, p. 181], we have that if $\left\{g\left(\xi_{k}\right)\right\}$ are linearly independent over $Q$, then

$$
\sup _{x \in R}\left|\sum a_{k} e^{i g\left(\xi_{k}\right) x}\right|=\sum\left|a_{k}\right|
$$

whence,

But

$$
\sum\left|a_{k}\right|=\left\|T^{*}\left(\sum a_{k} \Phi_{\phi_{\xi_{k}}}\right)\right\| \leq\left\|T^{*}\right\|\left\|\sum a_{k} \Phi_{\phi_{\xi_{k}}}\right\|
$$

$$
\left\|\sum a_{k} \Phi_{\phi_{\xi_{k}}}\right\|=\sup _{x \in R}\left|\sum a_{k} e^{i \xi_{k} x}\right|
$$

(Phragmén-Lindelöf again). Thus, if $\xi_{k} \in[-1,1]$ and $\left\{g\left(\xi_{k}\right)\right\}$ are linearly independent over $Q$, then

$$
\sum\left|a_{k}\right| \leq\left\|T^{*}\right\| \sup _{x \in R}\left|\sum a_{k} e^{i \xi_{k} x}\right|
$$

for all $a_{k} \in C$ and $\xi_{k} \in[-1,1]$. 
From here we observe that the argument used in the "first step" of the proof of the Beurling-Helson Theorem in [3, p. 217] carries over to our case. As a consequence, we can conclude that there is an interval $I \subset[-1,1]$ on which $g$ is a polynomial function. Finally, if we let $\tau$ be a non-constant linear function mapping $[-1,1]$ into $I$, then $g \circ \tau$ is a polynomial function on $[-1,1]$, and $g \circ \tau$ induces an endomorphism of $E a[-1,1]$. (This endomorphism is just the product $T T_{\tau}$, where $T_{\tau} f(x)=f(\tau(x))$.) By Lemma 2, $g \circ \tau$ must be linear on $[-1,1]$, and so $g$ is linear on $I$ as claimed.

We have thus shown that $g$ is made up of pieces of (possibly different) linear functions. Our final task will be to show that each $g$ which induces an endomorphism of $E a[-1,1]$ is linear on all of $[-1,1]$.

THEOREM. Suppose $T$ is an endomorphism of $E a[-1,1]$ and $T f(x)=f(g(x))$ for all $f \in E a[-1,1]$ and $x \in[-1,1]$. Then $g$ is a linear function on $[-1,1]$ of the form $g(\xi)=A \xi+B$, where $|A|+|B| \leq 1$.

ProOF. Again let $T$ and $g$ be as described in the hypotheses. The previous lemma showed that $g$ has the form $g(\xi)=A \xi+B$ on some interval $I \subset[-1,1]$. Let $k \geq 1$ be an integer, and let $\mu_{k}^{*}$ be a Borel measure in $M^{\omega}(C)$ with

$$
T\left(e^{i k u}\right)=e^{i k g(\xi)}=\int_{C} e^{\xi \lambda} d \mu_{k}^{*}(\lambda) .
$$

Since

$$
\left\|e^{i k g(\xi)}\right\|_{E a[-1,1]} \leq\|T\|\left\|e^{i k u}\right\|=\|T\|,
$$

we may assume that the measures $\mu_{k}^{*}$ satisfy

$$
\int_{C} e^{|\operatorname{Re} \lambda|} d\left|\mu_{k}^{*}\right|(\lambda) \leq 2\|T\|
$$

for all $k \geq 1$. For each $k \geq 1$, let $\mu_{k}(\lambda)=e^{-i k B} \mu_{k}^{*}(\lambda+i k A)$. Since $\int_{C} e^{|\operatorname{Re} \lambda|} d\left|\mu_{k}\right|(\lambda)$ $=\int_{C} e^{|\operatorname{Re} \lambda|} d\left|\mu_{k}^{*}\right|(\lambda),\left\{e^{|\operatorname{Re} \lambda|} d \mu_{k}\right\}$ and $\left\{d \mu_{k}\right\}$ are uniformly bounded sequences of regular Borel measures on $C$. In addition,

$$
\int_{C} e^{\xi \lambda} d \mu_{k}(\lambda)=e^{-i(A k \xi+B k)} \int_{C} e^{\xi \lambda} d \mu_{k}^{*}(\lambda)=e^{i[k g(\xi)-k(A \xi+B)]} .
$$

If we define $g_{1}$ by $g_{1}(\xi)=g(\xi)-(A \xi+B)$, then $g_{1}(\xi)=0$ when $g(\xi)=A \xi+B$ and $\int_{C} e^{\xi \lambda} d \mu_{k}(\lambda)=e^{i k g_{1}(\xi)}$.

Next, for each positive integer $n$, let the measure $\nu_{n}$ be defined by

$$
\nu_{n}=\frac{1}{2^{n}} \sum_{k=0}^{n}\left(\begin{array}{l}
n \\
k
\end{array}\right) \mu_{k},
$$

where $\mu_{0}$ is evaluation at 0 . Then, for each such $n, \nu_{n}$ is a Borel measure on $C$ and

$$
\int_{C} e^{|\operatorname{Re} \lambda|} d\left|\nu_{n}\right|(\lambda) \leq \frac{1}{2^{n}} \sum_{k=0}^{n}\left(\begin{array}{l}
n \\
k
\end{array}\right) \int_{C} e^{|\operatorname{Re} \lambda|} d\left|\mu_{k}\right|(\lambda) \leq 2\|T\| .
$$

Hence $\hat{\nu}_{n}(\xi)=\int_{C} e^{\xi \lambda} d \nu_{n}(\lambda)$ (as a Gelfand transform) is in $E a[-1,1]$, and also $\left\{\hat{\nu}_{n}\right\}$ is a bounded subset of $C[-1,1]$ in sup norm. Since $M(C)$ is the dual space of $C_{0}(C)$, the continuous complex-valued functions on $C$ which vanish at $\infty$, and 
since $\left\{e^{|\operatorname{Re} \lambda|} d \nu_{n}(\lambda)\right\}$ is a bounded sequence in $M(C)$, then, using subsequences if necessary, there exists a Borel measure $\nu$ on $C$ such that

$$
\int_{C} h(\lambda) e^{|\operatorname{Re} \lambda|} d \nu_{n}(\lambda) \rightarrow \int_{C} h(\lambda) e^{|\operatorname{Re} \lambda|} d \nu(\lambda)
$$

and

$$
\int_{C} h(\lambda) d \nu_{n}(\lambda) \rightarrow \int_{C} h(\lambda) d \nu(\lambda)
$$

for all $h \in C_{0}(C)$.

For $-1 \leq a<b \leq 1$, let $\Psi_{[a, b]}$ denote the characteristic function of the interval $[a, b]$, and let

$$
\begin{aligned}
h(\lambda) & =\left[\int_{-1}^{1} \Psi_{[a, b]}(\xi) e^{\xi \lambda} d \xi\right] e^{-|\operatorname{Re} \lambda|} \\
& =\left[\frac{e^{b \lambda}-e^{\alpha \lambda}}{\lambda}\right] e^{-|\operatorname{Re} \lambda|} .
\end{aligned}
$$

As a function of $\lambda, h$ belongs to $C_{0}(C)$, and a routine calculation shows that

$$
\int_{-1}^{1} \Psi_{[a, b]}(\xi) \hat{\nu}_{n}(\xi) d \xi \rightarrow \int_{-1}^{1} \Psi_{[a, b]}(\xi) \hat{\nu}(\xi) d \xi
$$

for all $a, b$ with $-1 \leq a<b \leq 1$.

Further,

$$
\begin{aligned}
\hat{\nu}_{n}(\xi) & =\int_{C} e^{\xi \lambda} d \nu_{n}(\lambda) \\
& =\frac{1}{2^{n}} \sum_{k=0}^{n}\left(\begin{array}{l}
n \\
k
\end{array}\right) \int_{C} e^{\xi \lambda} d \mu_{k}(\lambda) \\
& =\frac{1}{2^{n}} \sum_{k=0}^{n}\left(\begin{array}{l}
n \\
k
\end{array}\right) e^{i k g_{1}(\xi)} \\
& =\left(\frac{1+e^{i g_{1}(\xi)}}{2}\right)^{n}
\end{aligned}
$$

If we set $J=\left\{\xi \mid g_{1}(\xi)=0\right\}$, and let $\Psi_{J}$ denote the characteristic function of $J$, then $\hat{\nu}_{n}(\xi) \rightarrow \Psi_{J}(\xi)$ for all $\xi \in[-1,1]$. Also, since $\left\|\hat{\nu}_{n}\right\|_{\infty} \leq 2\|T\|$, the Lebesgue Dominated Convergence Theorem implies that

$$
\int_{-1}^{1} \Psi_{[a, b]}(\xi) \hat{\nu}_{n}(\xi) d \xi \rightarrow \int_{-1}^{1} \Psi_{[a, b]}(\xi) \Psi_{J}(\xi) d \xi
$$

for all $a, b$, with $-1 \leq a<b \leq 1$.

By combining (3) and (4), we conclude that

$$
\int_{-1}^{1} \Psi_{[a, b]}(\xi) \hat{\nu}(\xi) d \xi=\int_{-1}^{1} \Psi_{[a, b]}(\xi) \Psi_{J}(\xi) d \xi
$$

for all $a, b$. Thus, $\hat{\nu}(\xi)=\Psi_{J}(\xi)$ a.e. But $\hat{\nu}$ is continuous and the measure of $J \geq$ measure of $I>0$. Therefore, $\hat{\nu}(\xi)=1$ for all $\xi \in[-1,1]$, so that $J=$ $\left\{\xi \mid g_{1}(\xi)=0\right\}=[-1,1]$. That is, $g_{1}(\xi)=0$ on $[-1,1]$, and thus, $g(\xi)=A \xi+B$ 
on $[-1,1]$. Finally, since $g:[-1,1] \rightarrow[-1,1]$, it is easy to see that $|A|+|B| \leq 1$, which completes the proof of the Theorem.

We observe, too, that an endomorphism $T$ is an automorphism if, and only if, $B=0$ and $A=1$ or -1 .

As a final remark, the referee noted that since $E a[-1,1]$ is isometrically isomorphic to a quotient of $l^{1}(R)$, the $L^{1}$ group algebra of the discrete additive group of reals $[4$, p. 685], it is natural to ask which other quotients of group algebras also have the endomorphism property associated with the Beurling-Helson Theorem.

\section{REFERENCES}

1. F. F. Bonsall and J. Duncan, Numerical ranges. II, Cambridge Univ. Press, London, 1973.

2. Y. Domar, On the Banach algebra $A(\Gamma)$ for smooth sets $\Gamma \subset R^{n}$, Comment. Math. Helv. 52 (1977), 357-371.

3. Y. Katznelson, An introduction to harmonic analysis, Wiley, New York, 1968.

4. A. M. Sinclair, The Banach algebra generated by a hermitian element, Proc. London Math. Soc. (3) 24 (1972), 681-691.

Department OF MAThematics, University of MASSAChUSETtS at Boston, DORCHESTER, MASSACHUSETTS 02125 